\title{
Charting the survival, health and development of extremely preterm infants: EPICure and beyond
}

\author{
Samantha Johnson ${ }^{1}$ PhD, CPsychol, AFBPsS and Neil Marlow ${ }^{2}$ DM, FMedSci. \\ ${ }^{1}$ Department of Health Sciences, University of Leicester, Leicester, UK; \\ ${ }^{2}$ Institute for Women's Health, University College London, London, UK.
}

Corresponding author: Dr Samantha Johnson, Reader in Developmental Psychology, University of Leicester, Department of Health Sciences, Centre for Medicine, University Road, LE1 7RH, Email: sjj19@leicester.ac.uk, Tel: 0116252 5798, Fax: 01162523272.

Keywords: EPICure; extremely preterm: survival; outcomes; neurodevelopment

Word count: 3696

No of tables: 1

No of figures: 4

Funding: Neil Marlow receives part funding from the Department of Health's NIHR Biomedical Research Centre's funding scheme at UCLH/UCL.

Conflict of Interest: The authors have no conflicts of interest to disclose.

Abbreviations: Bayley-3 Bayley Scales of Infant and Toddler Development $3^{\text {rd }}$ Edition; BPD Bronchopulmonary Dysplasia; BSID-II Bayley Scales of Infant Development $2^{\text {nd }}$ Edition; CP Cerebral Palsy; EP Extremely preterm; IQ Intelligence Quotient; K-ABC Kaufman Assessment Battery for Children; MDI Mental Development Index; MPC Mental Processing Composite; NEC Necrotising enterocolitis; ROP Retinopathy of Prematurity 


\section{Introduction}

Major technological advances and improvements in neonatal medicine in the 1970s and 1980s resulted in significant reductions in mortality rates for babies born at extremely low gestations. In 1992, the gestational age for defining stillbirth in the UK was reduced from 28 weeks to 24 weeks reflecting the marked increase in survival for extremely preterm births. However, as the 'limits of viability' were pushed back ever further, there was increasing recognition of the high risk for residual disability amongst extremely preterm survivors. By the mid-1990s, clinicians were aware that the landscape had changed considerably for extremely preterm infants, but wide variation in survival rates between single centres and a lack of standardised developmental follow-up meant that it was difficult to gauge the impact of extremely preterm birth on a national level and to counsel parents as to the likely outcomes for their child. So in 1995, a team of neonatologists in the UK undertook the challenge of carrying out the first national epidemiological study of survival and outcomes following extremely preterm birth, and from that the EPICure Studies were born. Over the last 20 years these and other national cohort studies have helped shape neonatal care and advance our understanding of the life course consequences of extreme prematurity. Here we provide an overview of the key findings from the EPICure Studies and discuss the future challenges faced by clinicians and academics in tackling the causes, consequences and care of extremely preterm births.

\section{The EPICure Cohort}

Details of all babies born at $\leq 25$ weeks gestational age in each of the 276 maternity units in the whole of the UK and Republic of Ireland from $1^{\text {st }}$ March 1995 through $31^{\text {st }}$ December 1995 were collected prospectively (Table 1); these births formed the EPICure cohort. Initial data collection for all births comprised gestational age, whether signs of life were noted and whether the baby was admitted to a neonatal intensive care unit (NICU); for those admitted to NICU, a more detailed dataset was collected relating to the baby's neonatal course and maternal demographic information. At discharge from hospital, parental consent was sought to use these data and to invite the family to participate in follow-up evaluations.

To date, the EPICure cohort have been followed-up at 2.5 years, 6 years and 11 years of ag (Table 1) in order to determine rates of neurodevelopmental disability and other key outcomes. At 2.5 years of age, the outcome evaluation comprised a clinical assessment including a neurological examination, assessment of growth, hearing and vision, and a standardised developmental test (Bayley Scales of Infant Development 2nd Edition; BSID-II). At 6 and 11 years of age, a more complete evaluation was carried out that comprised a similar clinical assessment with additional psychometric tests and questionnaires to assess intelligence, behavioural, social, emotional and 
attention problems, psychiatric disorders and educational outcomes, and clinical tests to assess respiratory, cardiovascular and sensory outcomes.

A group of term-born controls was also assessed at 6 years $(n=160)$ and 11 years $(n=153)$ of age (Table 1). These comprised classmates of the EP children in mainstream schools who were matched for age, sex and ethnic group where possible, though these groups were ultimately smaller in number as controls could not be selected for every EP child. Importantly, there were no significant differences between EP children and controls in key confounding factors, such as age, sex and socioeconomic status at either 6 or 11 years, and rates of disorders among controls were as expected in the general population, thus providing a sound contemporary reference group.

\section{The EPICure-2 Cohort}

All births between 22 and 26 completed weeks gestational age (i.e. to 26 weeks and 6 days) were recorded from 182 maternity units in England in the calendar year from $1^{\text {st }}$ January 2006. Data collection was carried out in collaboration with the Centre for Maternal and Child Health Enquiries (CEMACH) and included enhanced perinatal data collection allowing for more detailed data on delivery room activity and interventions compared with the 1995 cohort. This provided information on fetal status when the woman was admitted to hospital for delivery and on the level of intervention after birth.

The population was offered follow up at 2.5-3 years of age, at which time we evaluated 576 (55\%) face to face and obtained detailed information from local neonatal data for a further 191 (18\%) children, giving a $74 \%$ follow up rate overall. We carried out a similar assessment to that in the original EPICure Study to allow comparison of neurodevelopmental outcomes over time. However, because of the changes in the structure of the $3^{\text {rd }}$ edition of the Bayley Scales (Bayley-3), we developed a formula to convert the new assessments to a BSID-II Mental Development Index (MDI) score comparable with the $2^{\text {nd }}$ edition of the test used in the 1995 cohort No controls were selected at this age.

\section{Survival and neonatal morbidities}

In 1995, we identified 4004 births between 20 and 25 completed weeks of gestation, with an overall survival of $39 \%$. Because of the way in which data were collected, information obtained before admission to the neonatal unit was not considered reliable so outcome data were ultimately based on 811 admissions for neonatal care. 
In 2006, from 3133 births at 22 to 26 weeks of gestation inclusive, we identified 2034 live births with an overall survival of $51 \%$ of live births. Considering births at 22 to 25 weeks gestation in the 10 month period from March through December in England only in 1995 and births in the same period in England in 2006, we identified a $44 \%$ increase in the number of admissions for neonatal care, from 666 in 1995 to 959 in 2006. There was also an increase in the use of antenatal steroids and improved early condition reflected in a greater proportion with heart rate $>100 \mathrm{bpm}$ at 5 minutes, admission temperature $>35^{\circ} \mathrm{C}$ and lower scores using the CRIB-1 score. In 2006 we were also able to identify those babies who did not receive active resuscitation in the delivery room allowing us to report survival for those for whom optimal intervention was provided, figures that more truly represent the outcome for counselling purposes.

Improved survival in 2006 reflected greater numbers of babies surviving the first week after birth, which was mirrored in more deaths being ascribed to later complications of prematurity. Disappointingly, there was little difference in the pattern of neonatal morbidities - the proportion of survivors with Bronchopulmonary Dysplasia (BPD) and with brain injuries were similar in the two cohorts, growth in weight and in head circumference was similar and the proportion discharged home in oxygen rose by $7 \%$ ( $0.2 \%$ in 1995 to $14 \%$ in 2006 ) and with retinal surgery by $8 \%$ ( $3 \%$ in 1995 to $13 \%$ in 2006). Survival without neonatal morbidities in 2006 was 23 weeks: $23 \%$ (95\% Cl 13, 35); 24 weeks: $29 \%$ (95\% Cl 23, 27); 25 weeks: $38 \%$ (95\% Cl 33, 44); and 26 weeks 50\% (95\% Cl 45, 54) (Figure 1).

\section{Neurodevelopmental disability}

EPICure: Of the core neurodevelopmental outcomes assessed at 2.5, 6 and 11 years of age in the 1995 cohort, developmental delays and intellectual disabilities were far more common than neuromotor or sensory disabilities (Table 1). When assessed at 2.5 years of age, less than $10 \%$ of EP survivors had severe cerebral palsy (CP), vision or hearing impairments (Table 1); however, $19 \%$ had developmental test scores in the severely delayed range (scores $<-3 \mathrm{SD}$ ) and a further $11 \% \mathrm{had}$ moderately delayed development (scores -2 to $-3 \mathrm{SD}$ ). As neurodevelopmental impairments frequently co-exist, the most severe rating in terms of development, neuromotor, vision or hearing impairment was used to classify severity of neurodevelopmental disability. Using this commonly reported composite outcome, $23 \%$ had severe disability and a further $25 \%$ had moderate disability, resulting in $49 \%$ prevalence of neurodevelopmental disability overall at 2.5 years of age (Figure 2).

EPICure-2: In the 2006 cohort, early childhood outcomes were assessed at 3 years of age on average. Although follow-up rates for face to face assessments were low (Table 1), data were available to 
classify impairment in $74 \%$ of the cohort. Imputing results for all children who did not have a face-toface assessment did not alter the findings and regression analyses accounting for the selective drop out of families from more disadvantaged socio-economic groups did not alter the findings significantly. Overall, $25 \%$ were categorised with moderate or severe impairment, which equated to $45 \%$ at 22 to 23 weeks, $30 \%$ at 24 weeks, $25 \%$ at 25 weeks and $20 \%$ at 26 weeks gestation. A similar pattern of impairment was observed, with the majority of children classified as moderate or severely impaired because of low cognitive scores. After conversion of the Bayley-3 cognitive and language test scores to MDI equivalent scores for comparison to the 1995 cohort, mean scores were 80 (SD 21) at 22 to 23 weeks, 87 (SD 19) at 24 weeks, 88 (SD 19) at 25 weeks and 91 (SD 18) at 26 weeks of gestation. Between 22 and 25 weeks gestation there was an average improvement in mean scores of $8 \mathrm{MDI}$ points between the two cohorts. Severe cerebral palsy (Gross Motor Function Classification System (GMFCS) grades III-V) occurred in a small proportion of survivors ( $9 \%$ at 22 to 23 weeks; $5 \%$ at 24 weeks; $4 \%$ at 25 weeks and $2 \%$ at 26 weeks). As in the first cohort, there were few children with sensory impairments in the EPICure-2 cohort.

Changes over time: Because the data in 1995 were validated for NICU admissions only, we compared the population of children born at 22 to 25 weeks gestation in English neonatal services in 1995 and in 2006, with outcomes classified in the same manner. Overall results are shown in Figure 3 , demonstrating a steady increase in survival without neurodevelopmental disability at each gestational week. The change in the proportion of NICU admissions categorised with severe (or moderate) disability at each gestational week was not significant and indicated a small increase, but this was offset by a much larger increase in the chances of surviving without moderate or severe disability between 1995 and 2006, which was significant overall and at 24 and 25 weeks of gestation.

Changes over childhood: Evaluating children in the 1995 EPICure cohort at all three ages led to changes in classification of neurodevelopmental disability for many children at different time points, but overall the proportions with moderate and severe disabilities remained fairly constant (Figure 3 ). Imputing outcomes to account for children lost to follow-up between 2.5 and 11 years indicated that, over childhood, approximately $50 \%$ were classified with severe or moderate disability, split approximately $50: 50$ between the two categories (Figure 3 ).

At all ages, rates of neuromotor and sensory impairments were markedly lower than intellectual impairments (Table 1). In terms of general cognitive measures, the Mean BSID-II MDI score at 2.5 years was 84 (SD 12), which was approximately 1 SD below the normative test mean of 100. At 6 and 11 years, deficits of 1.3 SD to 1.6 SD were observed using the mental processing composite (MPC; IQ equivalent) score of the Kaufman Assessment Battery for Children (K-ABC) when compared to 
contemporaneous reference data (6 years: mean difference - 24 points, $95 \% \mathrm{Cl}-27,-20 ; 11$ years: 20 points, $95 \% \mathrm{Cl}-23,-17)$. Notably, the rate of moderate to severe intellectual impairment (scores < -2 SD of the control group) was remarkably similar over time, with $41 \%$ at 6 years and $40 \%$ at 11 years of age, compared with $1.3 \%$ of term born controls ( 6 years: OR 56, 95\% Cl 13, 250; 11 years: OR $50,95 \% \mathrm{Cl} 12,206)$. At both ages, the deficit was greater in simultaneous rather than sequential information processing, indicating that EP children have greatest difficulty in integrating complex information from multiple sources.

At all ages boys fared worse than girls with boys having higher rates of neurodevelopmental disability and significantly lower $\mathrm{IQ} /$ developmental test scores. There was also a trend for poorer outcomes in those born at 22 to 24 weeks than in those born at 25 weeks, but associations with gestational age are difficult to assess given the restricted range of gestations studied and the small number of infants born at 22 to 23 weeks. Notably, although there was substantial variation in developmental trajectories making the predictive validity of early assessments relatively poor at the individual level, the stability in the prevalence of moderate and severe disability over childhood (Figure 3) make these data useful for estimating the impact of EP birth on a population level and to support the counselling of parents regarding the likely outcomes for their EP child.

\section{Psychological and educational outcomes}

As the children matured there was scope for assessing a wider range of outcomes and at 6 and 11 years greater focus was placed on assessing the impact of EP birth on psychological and educational outcomes in the first EPICure cohort. At 6 years of age, using agreement between parent- and teacher-reports, $19.4 \%$ of EP children were rated with clinically significant behaviour problems compared with $3.4 \%$ of term-born controls (OR $6.8,95 \% \mathrm{Cl} 2.6,17.9)$. After adjustment for IQ, there was no significant excess of hyperactivity or conduct problems, but there remained a 2 to 4 times increase in the odds of clinically significant emotional problems (OR $4.2,95 \% \mathrm{Cl} 1.5,11.8$ ), social problems (OR 2.5, 95\% $\mathrm{Cl} 1.0,6.1$ ) and attention problems (OR 3.8, 95\% $\mathrm{Cl} 1.7,8 ., 3$ ) among the $\mathrm{EP}$ children. A similar pattern of results was observed at 11 years of age when the prevalence of psychiatric disorders was assessed for the first time using a diagnostic evaluation, on which $23 \%$ of EP children and $9 \%$ of classmates had psychiatric diagnoses (OR 3.2, 95\% $\mathrm{Cl} 1.7,6.2$ ). The increased risk was specific to emotional disorders ( $9 \%$ vs. $2 \%$, OR $4.6,95 \% \mathrm{Cl} 1.3,15.9$ ), autism spectrum disorders ( $8 \%$ vs. $0 \%, \mathrm{p}<0.001)$ and $\mathrm{ADHD}(11.5 \%$ vs. $3 \%, \mathrm{OR} 4.3,95 \% \mathrm{Cl} 1.5,13.0)$. Parent and teacher reported attention and conduct problems at 6 years of age were significant predictors of a psychiatric disorder at 11 years, indicating continuity in outcomes and the persistence of psychiatric 
sequelae in this population. In addition to those with psychiatric disorders, many more children were rated by their parents or teachers as having clinically significant attention, social or emotional problems, and therefore the demands on healthcare and education services for long-term support may be underestimated by focusing only on those who meet diagnostic criteria.

The high rate of cognitive impairment and behavioural sequelae in this population no doubt impacts on EP children's learning and attainment at school. It was therefore little surprise that at both 6 and 11 years of age, $50 \%$ of EP children were rated by their teachers as having attainment below the national average compared with $5 \%$ of their term-born classmates (6 years: OR 17.9, 95\% $\mathrm{Cl} 8.3$, 38.9; 11 years: $\mathrm{OR} 18.2,95 \% \mathrm{Cl} 8.0,41.4)$. Among children in mainstream schools, the relative deficit in attainment between EP children and controls was greater in mathematics compared with English/Literacy and other subjects (Figure 4). This was also observed on psychometric testing at 11 years of age in which $30 \%$ of EP children were classified with learning difficulties in reading compared with $2 \%$ of controls ( $\mathrm{OR} 22,95 \% \mathrm{Cl} 6.6,70$ ), and $44 \%$ with mathematics learning difficulties compared with $1.3 \%$ of controls (OR $59,95 \% \mathrm{Cl} 14,243$ ). At this age, $13 \%$ attended a special school and, of those in mainstream schools, $57 \%$ had special educational needs compared with $11 \%$ of classmates (OR $10.3,95 \% \mathrm{Cl} 5.8,18.4)$. In health economic analyses, costs associated with EP birth were significantly higher than for term-born children over the $11^{\text {th }}$ year of life, with a mean cost increase of $£ 2477(95 \% \mathrm{Cl} £ 1605, £ 3360)$ in public sector costs, with the greatest costs associated with education rather than healthcare or social services.

\section{Other important outcomes}

Despite the focus on neurodevelopmental morbidity, prematurity has a pervasive effect on the development of other organ systems, many of which are not yet characterised well. Given the high rate of neonatal respiratory morbidity, it is not surprising that lung function testing in early adolescence revealed a significant reduction in $\mathrm{FEV}_{1}$ and greater reversibility following bronchodilators among the EP group compared with their term-born controls in the 1995 cohort. This pattern of impairment has implications for adult prognosis and studies are underway to characterise the adult deficit. In parallel with this, we have demonstrated small increases in blood pressure and a significant increase in augmentation index on vascular assessment which too may have implications for adult cardiovascular disease. Of note, pulse wave velocity in the main arteries was similar in the two groups implying that the cause of the increased augmentation pressure lay within the distal circulation and not a reduction in elasticity of the major vessels. At the 11-year assessment there was an association between $\mathrm{FEV}_{1}$ and augmentation index, leading to speculation that they might share a similar aetiology, such as a reduced tissue elastin content. Much more work 
is needed to establish the cardiorespiratory and associated metabolic risk for this group, alongside the characterisation of the effects of EP birth on other organ systems, such as the kidney.

\section{The EPICure Studies: An international perspective}

Survival at extremely low gestational ages is heavily influenced by the local attitude of professionals to resuscitation and by the willingness of doctors to optimise the conditions of delivery, with variation being widest at 22 and 23 weeks of gestation. Besides variation between countries, there is also much variation in practice between individual hospitals, for example within the National Institute of Child Health and Human Development (NICHD) network in the USA, within Swedish regions, and within different categories of neonatal services, making direct comparisons difficult to make.

Considering the prevalence of impairments among survivors, again definitions of the baseline population and individual outcomes may also vary. Similar rates of impairment in 2.5 to 3 year neurodevelopmental outcomes were found between EP infants in the Swedish EXPRESS Study and the EPICure-2 cohort despite very different survival rates. As with studies of cerebral palsy over the period when intensive care was introduced, neurodevelopmental morbidity rates may be difficult to reduce over a period of increasing survival.

\section{Beyond EPICure}

In 1995, EPICure was the largest study of extreme prematurity in the world and, since publication of the first data in 2000 , has contributed to our understanding of the factors affecting survival at extremely low gestations, the characterisation of the extremely preterm phenotype and how changes in neonatal care have impacted on survival and outcomes over time. However, whilst we know more than ever before, significant challenges remain for researchers worldwide. These include:

- Charting lifelong outcomes. Reports of outcomes into adulthood for the earliest EP cohorts are beginning to emerge, and publications from the latest follow-up of the EPICure cohort at 19 years of age are imminent. An exploration of outcomes throughout adulthood is needed to truly understand the life course consequences of EP birth and how early deficits evolve over the lifespan. Studies of VLBW cohorts from the 1970s and 1980s suggest that neurodevelopmental, cognitive and psychiatric problems persist into adulthood with a similar magnitude, but that outcomes might be better than once expected with the majority having a good quality of life. However as these cohorts include relatively few adults born $<26$ weeks of gestation, 
contemporary data are needed to understand what life is likely to bring for EP survivors today. Furthermore, other important outcomes such as respiratory and cardiovascular function are little studied during transition to adult life and the interaction between prematurity and ageing has received little attention as yet.

- Refining neonatal care. Continued investment in longitudinal studies are needed to determine how survival and long term outcomes change over time in relation to changes in neonatal care. To add to this literature, follow-up of the EPICure-2 cohort at 11 years of age will commence in 2017 enabling us to assess how changes in neonatal care between 1995 and 2006 have impacted on outcomes in middle childhood and whether this differs from the impact on developmental outcomes observed at 3 years of age. Understanding how new perinatal interventions and the organisation and delivery of neonatal care impacts on survival and short term morbidity is key in refining neonatal services and in improving long term quality of life.

- Improving developmental surveillance. Despite changes in neonatal care, EP infants remain at high risk for a range of developmental problems and disorders. To date there has been little investment in developmental surveillance for EP infants and there is wide variation in the structure and content of neonatal follow-up services across the UK. Follow-up is essential to ensure that children with significant difficulties are identified and provided with appropriate and timely health or social care. Future work should consider how long-term support can best be provided for EP children and their families.

- Elucidating aetiological mechanisms. EP birth confers a multiplicity of interrelated biological, neonatal and social risk factors. Elucidating mediating factors and specific aetiological mechanisms that underpin adverse outcomes are needed in order guide the development of new therapies and to identify targets for intervention.

- Developing effective intervention strategies. The ongoing development and evaluation of perinatal interventions and neuroprotective therapies is needed to attenuate the long term effects of extreme prematurity and concomitant intensive care on infants' health and development. There is also growing interest in the development of interventions for improving learning, cognition and behavioural outcomes at school age. Whether the most effective approach will be to develop population-specific interventions or to evaluate the efficacy of existing strategies in the EP population remains to be seen.

- Supporting collaborative research. Although preterm birth rates are increasing, EP birth still affects only $1-2 \%$ of all pregnancies. Establishing large cohorts thus necessitates substantial resources. Combining the efforts of investigators throughout the world to develop core datasets and to share and harmonise data will aid in increasing the statistical power of cohort studies for 
investigating the effect of EP birth on rare outcomes, identifying the effects of different healthcare systems, policies and approaches to perinatal care, and discovering universal mechanisms that contribute to the mortality and morbidity associated with EP birth.

\section{Further reading}

Costeloe K, Hennessy E, Gibson A, Marlow N, Wilkinson A. The EPICure Study: Outcomes to discharge from hospital for infants born at the threshold of viability. Pediatrics 2000;106:659-71.

Costeloe KL, Hennessy EM, Haider S, Stacey F, Marlow N, Draper ES. Short term outcomes after extreme preterm birth in England: comparison of two birth cohorts in 1995 and 2006. BMJ 2012;345:e7976.

Bolton CE, Stocks J, Hennessy E, et al. The EPICure study: association between hemodynamics and lung function at 11 years after extremely preterm birth. Journal of Pediatrics 2012;161:595-601.

Fawke J, Lum S, Kirkby J, et al. Lung function and respiratory symptoms at 11 years in children born extremely preterm: the EPICure study. American Journal of Respiratory and Critical Care Medicine 2010;182:237-45.

Johnson S, Fawke J, Hennessy E, et al. Neurodevelopmental disability through 11 years in children born before 26 weeks of gestation: The EPICure Study. Pediatrics 2009:e249-e57.

Johnson S, Hennessy E, Smith R, Trikic R, Wolke D, Marlow N. Academic attainment and special educational needs in extremely preterm children at 11 years. The EPICure Study. Archives of Disease in Childhood Fetal and Neonatal Edition 2009;94:F283-F9.

Johnson S, Hollis C, Kochhar P, Hennessy E, Wolke D, Marlow N. Psychiatric Disorders in Extremely Preterm Children: Longitudinal Finding at Age 11 Years in the EPICure Study. Journal of the American Academy of Child \& Adolescent Psychiatry 2010;49:453-63.

Marlow N, Wolke D, Bracewell MA, Samara M. Neurologic and developmental disability at six years of age after extremely preterm birth. New England Journal of Medicine 2005;352:9-19.

Marlow N, Bennett C, Draper ES, Hennessy EM, Morgan AS, Costeloe KL. Perinatal outcomes for extremely preterm babies in relation to place of birth in England: the EPICure 2 study. Archives of Disease in Childhood Fetal and Neonatal Edition 2014;99:F181-8.

McEniery CM, Bolton CE, Fawke J, et al. Cardiovascular consequences of extreme prematurity: the EPICure study. Journal of Hypertension 2011;29:1367-73.

Moore T, Hennessy EM, Myles J, et al. Neurological and developmental outcome in extremely preterm children born in England in 1995 and 2006: the EPICure studies. BMJ 2012;345:e7961.

Petrou S, Abangma G, Johnson S, Wolke D, Marlow N. Costs and health utilities associated with extremely preterm birth: evidence from the EPICure study. Value in Health 2009;12:1124-34. 
Samara M, Marlow N, Wolke D. Pervasive Behavior Problems at 6 Years of Age in a Total-Population Sample of Children Born at <=25 Weeks of Gestation. Pediatrics 2008;122:562-73.

Wolke D, Samara M, Bracewell M, Marlow N. Specific language difficulties and school achievement in children born at 25 weeks of gestation or less. Journal of Pediatrics 2008;152:256-62.

Wood NS, Marlow N, Costeloe K, Gibson AT, Wilkinson AR. Neurologic and developmental disability after extremely preterm birth. EPICure Study Group. N England Journal of Medicine 2000;343:37884. 
Table 1: Recruitment, follow-up rates and key neurodevelopmental outcomes of the EPICure (1995) and EPICure-2 (2006) cohorts.

\begin{tabular}{|c|c|c|c|}
\hline & $\begin{array}{c}\text { EPICure } \\
\text { UK, } \leq 25 \text { weeks } \\
\text { Mar-Dec } 1995\end{array}$ & $\begin{array}{c}\text { EPICure } \\
\text { Term-born } \\
\text { Controls }\end{array}$ & $\begin{array}{c}\text { EPICure-2 } \\
\text { England, } 22-26 w \\
2006\end{array}$ \\
\hline All births, $n$ & 4004 & - & 3133 \\
\hline Live births, $\mathrm{n}$ & 1289 & - & 2034 \\
\hline Births with active intervention, $\mathrm{n}$ & $n / k$ & - & 1842 \\
\hline Admitted to NICU, $\mathrm{n}$ & 811 & - & 1686 \\
\hline Survival to discharge, $\mathrm{n}$ & 314 & - & 1041 \\
\hline$\%$ of births with active intervention & $\mathrm{n} / \mathrm{k}$ & - & $56 \%$ \\
\hline$\%$ of NICU admissions & $39 \%$ & - & $61 \%$ \\
\hline Assessed at 2.5 years, $\mathrm{n}$ (\% of survivors at 2.5 years) & $283(92 \%)$ & - & $576(55.9 \%)$ \\
\hline Severe cerebral palsy, $\mathrm{n}$ (\% of survivors) & $27(9.5 \%)$ & - & $30(5.0 \%)$ \\
\hline Severe visual impairment, $\mathrm{n}$ (\% of survivors) & $7(2.5 \%)$ & - & $6(1.0 \%)$ \\
\hline Severe hearing impairment, $\mathrm{n}$ (\% of survivors) & $5(1.8 \%)$ & - & $1(0.2 \%)$ \\
\hline Severe developmental delay, $\mathrm{n}$ (\% of survivors) & $53(18.7 \%)$ & - & $57(9.9 \%)$ \\
\hline Any severe disability, $\mathrm{n}$ (\% of survivors) & $64(22.6 \%)$ & - & $77(13.4 \%)$ \\
\hline Assessed at 6 years, $n$ (\% of survivors at 6 years) & $241(78 \%)$ & 160 & - \\
\hline Severe cerebral palsy, $\mathrm{n}$ (\% of survivors) & $15(6.2 \%)$ & $0(0 \%)$ & - \\
\hline Severe visual impairment, $\mathrm{n}$ (\% of survivors) & $6(2.5 \%)$ & $0(0 \%)$ & - \\
\hline Severe hearing impairment, $\mathrm{n}$ (\% of survivors) & $7(2.9 \%)$ & $0(0 \%)$ & - \\
\hline Severe cognitive impairment, $\mathrm{n}$ (\% of survivors) & $50(20.7 \%)$ & $0(0 \%)$ & - \\
\hline Any severe disability, $\mathrm{n}$ (\% of survivors) & $53(21.9 \%)$ & $0(0 \%)$ & - \\
\hline Assessed at 11 years, $\mathrm{n}$ (\% of survivors at 11 years) & $219(71 \%)$ & 153 & - \\
\hline Severe cerebral palsy, $\mathrm{n}$ (\% of survivors) & $21(9.6 \%)$ & $0(0 \%)$ & - \\
\hline Severe visual impairment, $\mathrm{n}$ (\% of survivors) & $3(1.4 \%)$ & $0(0 \%)$ & - \\
\hline Severe hearing impairment, $\mathrm{n}$ (\% of survivors) & $1(0.5 \%)$ & $0(0 \%)$ & - \\
\hline Severe cognitive impairment, $\mathrm{n}$ (\% of survivors) & $32(14.6 \%)$ & $0(0 \%)$ & - \\
\hline Any severe disability, $\mathrm{n}$ (\% of survivors) & $34(15.5 \%)$ & $0(0 \%)$ & - \\
\hline
\end{tabular}

$\mathrm{n} / \mathrm{k}$ Not known. Severe visual impairment defined as blind or perceives light only. Severe hearing impairment defined as profound hearing loss not corrected with aids. Severe cognitive impairment at 2.5 years defined as Bayley Scales of Infant Development-II Mental Development Index Score <-3 SD of the normative population (score <55); severe cognitive impairment at 6 and 11 years defied as Kaufman Assessment Battery for Children Mental Processing Composite Score $<-3$ SD of the term-reference group (6 years MPC $<70 ; 11$ years MPC $<71$ ). Severe Cerebral Palsy is defined as Cerebral Palsy with severe functional impairment (i.e., non-ambulant (1995) or Gross Motor Function Classification Scale Levels III, IV or V (2006)). 


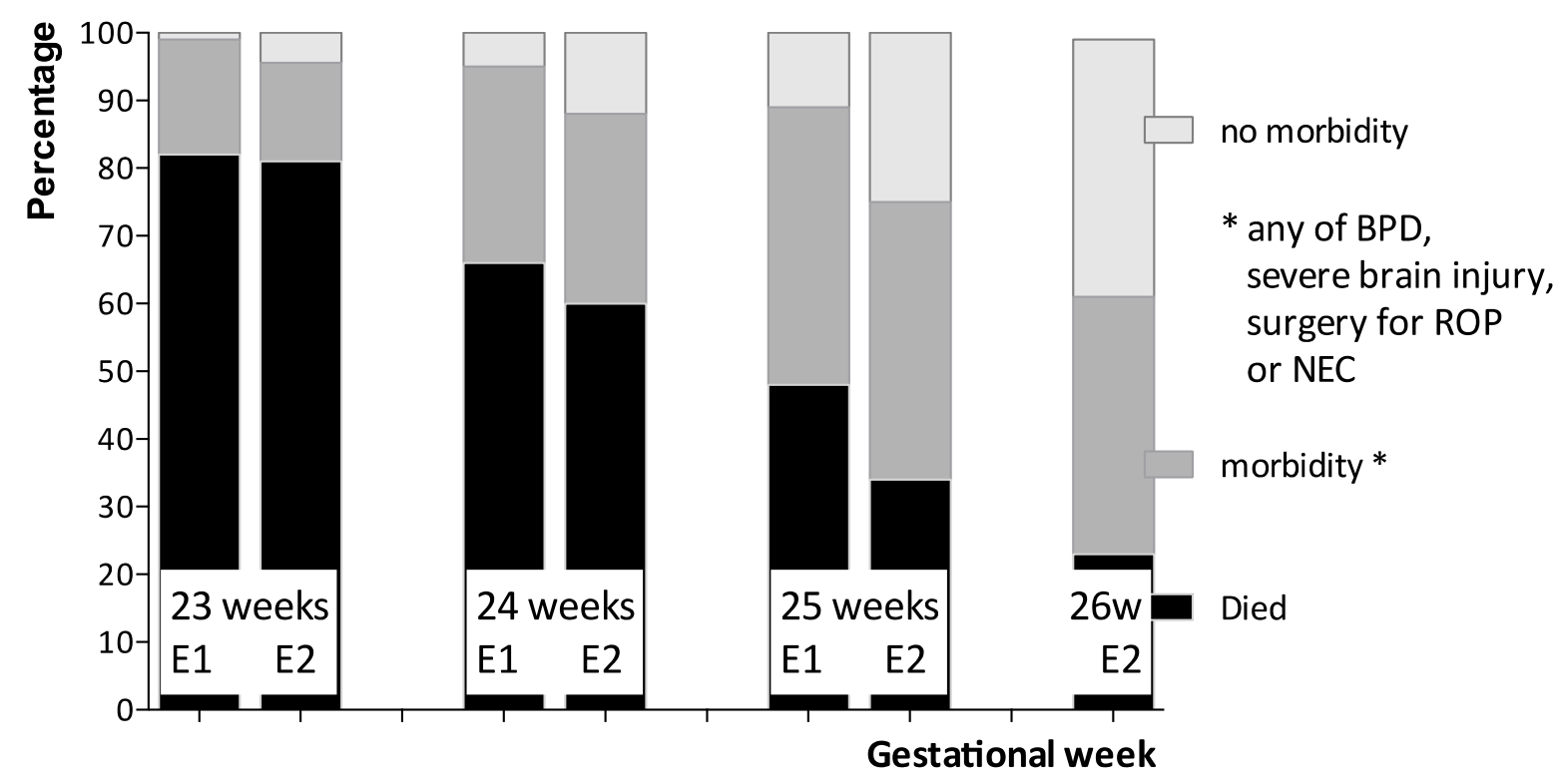

Figure 1. Survival with and without neonatal morbidity in EPICure (E1) and EPICure-2 (E2) by gestational week. Neonatal morbidity is any of bronchopulmonary dysplasia (BPD), severe brain injury, surgery for retinopathy of prematurity (ROP) or necrotising enterocolitis (NEC) 
Outcomes for babies admitted to NICU for care by gestational week

for the two EPICure studies (odds are shown for survival with severe disability

or no disability as a proportion of the admitted population).

Change between 1995 and 2006

\section{Gestation}

Severe disability No disability

22w 1995

2006

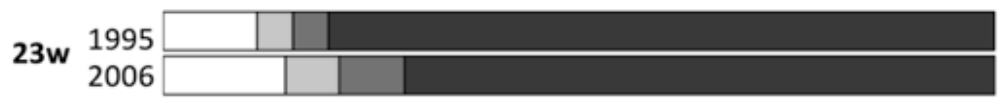

$1 \cdot 9(\cdot 67$ to $5 \cdot 2) \quad 1.4(\cdot 68$ to $2 \cdot 7)$

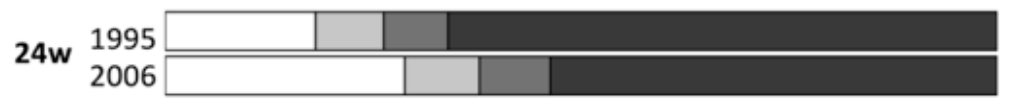

$1 \cdot 2(\cdot 68$ to $2 \cdot 1) \quad 1.8(1.2$ to $2 \cdot 7)$

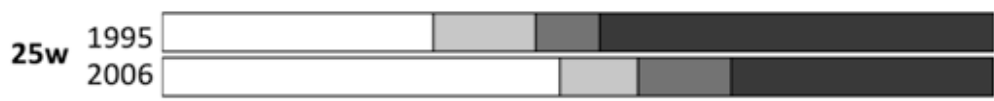

$1.4(\cdot 87$ to 2.4$) \quad 1.9(1.4$ to 2.6$)$

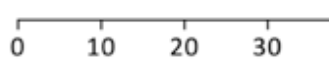

\section{Percentage (\%)}

No disability

Other disability

Severe disability

Died

Figure 2. Outcomes for babies admitted to NICU by gestational week for the two EPICure Studies. Odds ratios are shown for survival with severe disability or no disability as a proportion of the admitted population. 


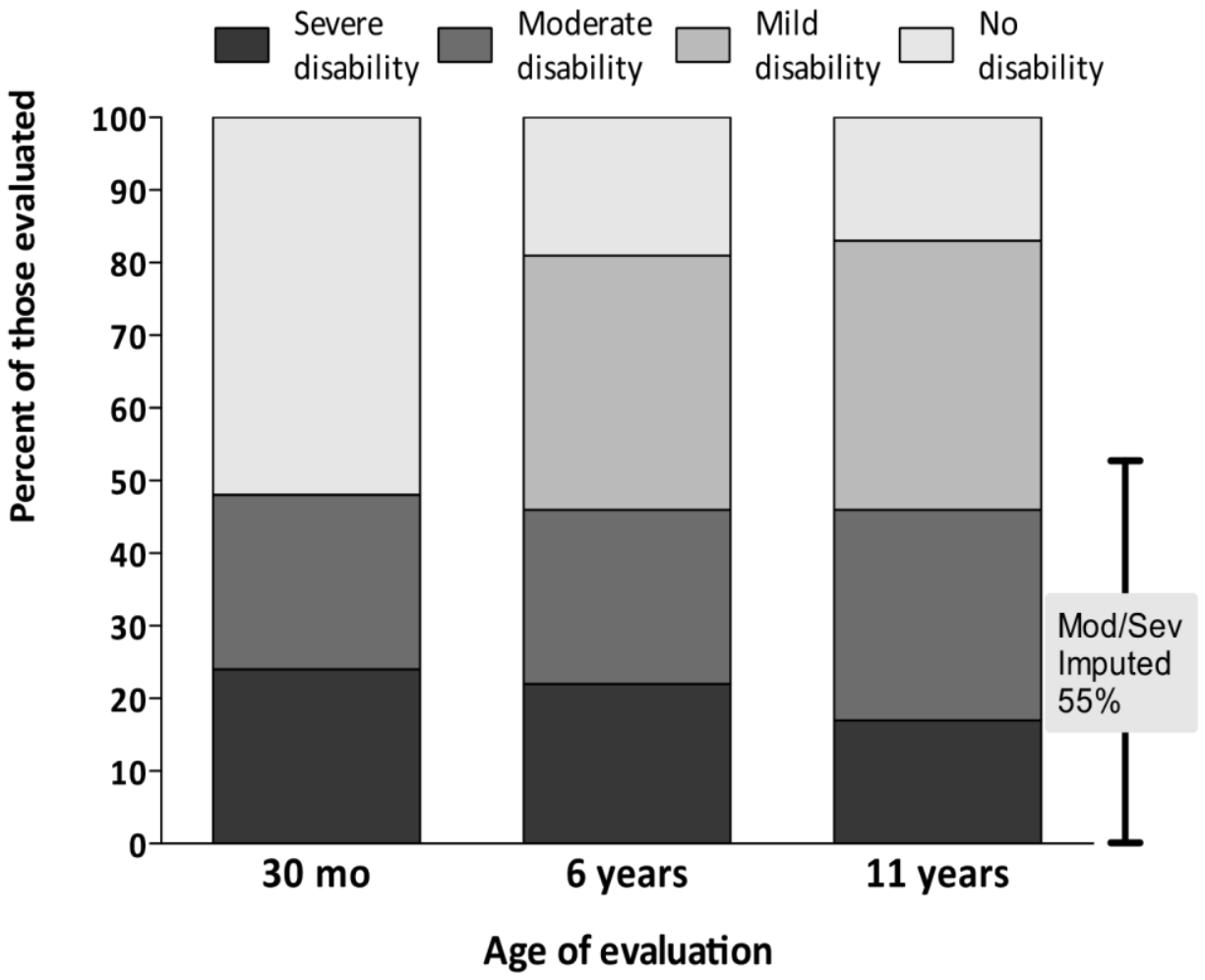

Figure 3. Proportion of extremely preterm survivors with neurodevelopmental disability evaluated at 2.5 years, 6 years and 11 years of age in the UK and Ireland EPICure Study of births $\leq 25$ weeks of gestation in 1995. (Outcome at 11 years is imputed to account for children lost to follow-up between 2.5 and 11 years of age.) 


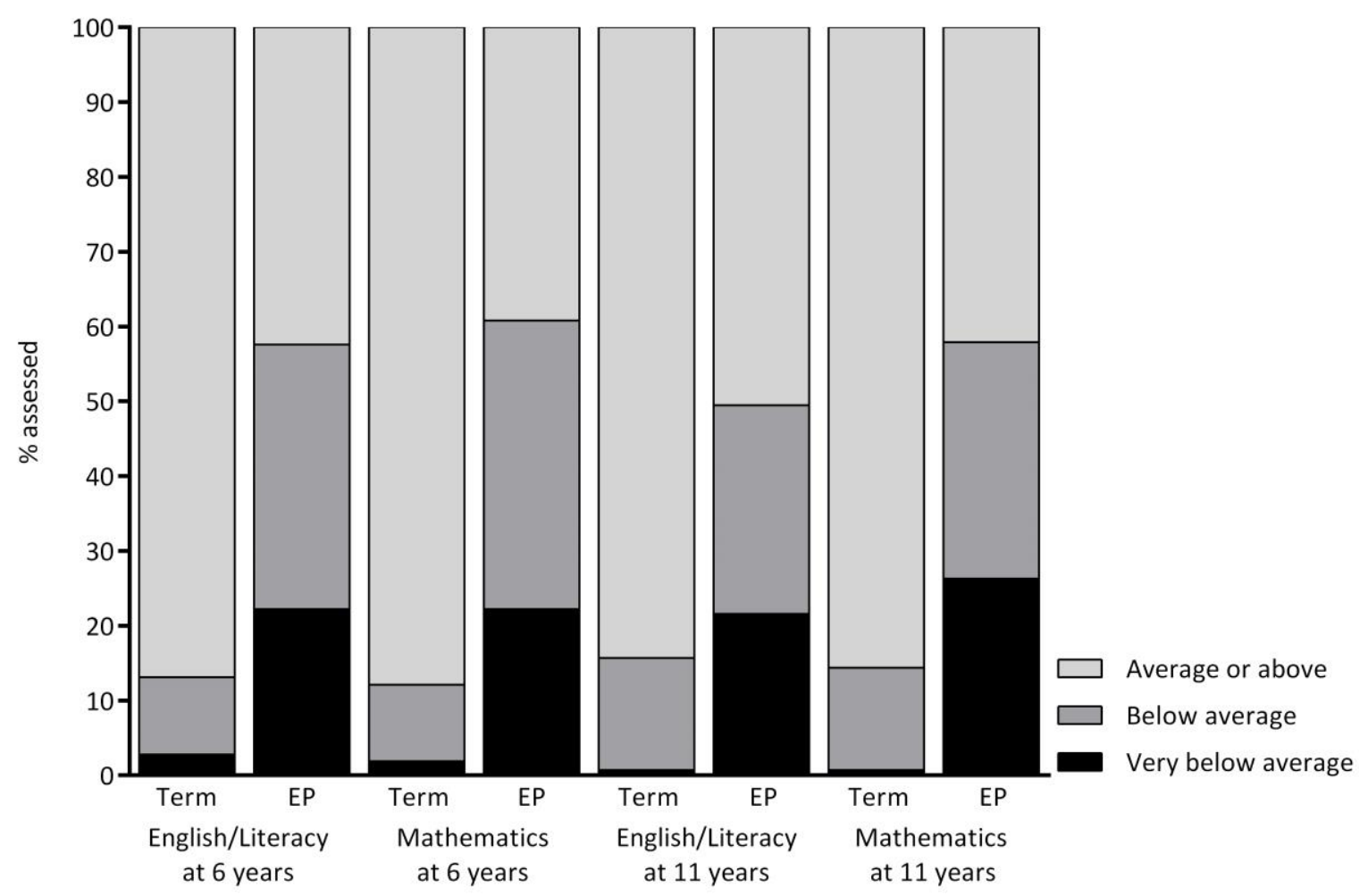

Figure 4. Academic attainment in relation to the national average expected for age as rated by class teachers for extremely preterm children and term-born controls in mainstream schools assessed at both 6 and 11 years of age in the UK and Ireland EPICure Study of births $\leq 25$ weeks of gestation in 1995. Bars represent the percent of children with each level of attainment by group. 